\title{
Antisemitismus der Anderen? - Einleitende Überlegungen
}

\author{
Nikolaus Hagen und Tobias Neuburger
}

Vor wenigen Jahren erschütterte eine tödliche Serie von Terroranschlägen Europa. Unter den Anschlagszielen befanden sich vor allem jüdische Einrichtungen (oder solche, die als „jüdisch“ wahrgenommen wurden), wie das Jüdische Museum Belgiens in Brüssel im Mai 2014, ${ }^{1}$ ein koscherer Supermarkt in Paris im Januar 2015 (zwei Tage nach dem Angriff auf die Redaktion des Satiremagazins Charlie Hebdo) ${ }^{2}$ und eine Synagoge in der dänischen Hauptstadt Kopenhagen im Monat darauf. ${ }^{3}$ Viele der Täter, darunter der Attentäter von Brüssel, bekannten sich offen zur dschihadistischen Gruppe „Islamischer Staat“ oder waren von deren Ideologie inspiriert. So sehr diese Anschläge allerdings die Öffentlichkeit schockierten: antisemitische Gewalt ist in Europa alles andere als eine Neuheit. Doch gerade die unheimliche Kontinuität des Antisemitismus gerät im Zuge der Skandalisierung allzu häufig aus dem Blick und wird geradezu durch sie verstellt.

Bereits in den 1970er-Jahren zielte der palästinensische Terrorismus ${ }^{4}$ - auch unter Beteiligung von Vertretern der europäischen Neuen Linken - auf jüdische und israelische Einrichtungen in Europa bzw. das, was die Terroristen für solche hielten. Besonders blutig war der Anschlag palästinensischer Terroristen auf die israelische Mannschaft bei den Olympischen Spielen 1972 in München, bei dem elf israelische Sportler und ein bayerischer Polizist starben. ${ }^{5}$ Niemals aufgeklärt wurde ein verheerender Brandanschlag, der sich zwei Jahre zuvor ebenfalls in München ereignet hatte: Am 13. Februar 1970 fielen sieben Shoah-Überlebende einem Brandanschlag auf das Jüdische Gemeindezentrum zum Opfer. Ermittelt wurde zu Beginn sowohl in rechten Kreisen als auch im Umfeld palästinensischer Terrorgruppen, im Anschluss auch im Umfeld linksterroristischer Organisationen - eine Aufklärung von Tat und Täterschaft konnten

\footnotetext{
${ }^{1}$ Gericht spricht Attentäter von Brüssel schuldig, in: Die Zeit, 7.3.2019, [https://www.zeit.de/gesellschaft/zeitgeschehen/2019-03/mehdi-nemmouche-anschlag-juedisches-museumbruessel-gericht-schuldig], eingesehen 1.2.2020.

2 Michael Thaidigsmann, Fünf Jahre nach dem Albtraum, in: Jüdische Allgemeine, 9.1.2020, [https://www.juedischeallgemeine.de/juedische-welt/ fuenf-jahre-nach-dem-albtraum/], eingesehen 1.2.2020.

3 Terror in Kopenhagen, in: Jüdische Allgemeine, 15.2.2015, [https://www.juedische-allgemeine.de/juedischewelt/terror-in-kopenhagen/], eingesehen 1.2.2020.

4 Siehe bspw. Thomas Riegler, Im Fadenkreuz: Österreich und der Nahostterrorismus 1973 bis 1985, Göttingen 2010; Ariel Merari/Shlomi Elad, The International Dimension of Palestinian Terrorism, New York 2019.

5 Eva Oberloskamp, Das Olympia-Attentat 1972. Politische Lernprozesse im Umgang mit dem transnationalen Terrorismus, in: Vierteljahrshefte für Zeitgeschichte 60 (2012), Heft 3, S. 321-352.
} 
auch Nachermittlungen der Bundesanwaltschaft, die 2017 ohne Ergebnis eingestellt wurden, nicht herstellen. ${ }^{6}$

Die gewalttätige Spur des Antisemitismus nach 1945 lässt sich auch nach Österreich verfolgen: Am jüdischen Neujahrsfest im September 1973 nahmen palästinensische Terroristen drei jüdische Geiseln am niederösterreichischen Grenzbahnhof Marchegg. Die Geiselnahme endete unblutig, zeitigte aber den gewünschten Erfolg: die österreichische Bundesregierung ging auf die Forderung der Täter ein und schloss ein Transitzentrum für jüdische Auswanderer aus der Sowjetunion. ${ }^{7}$ Im August 1981 war der israelitische Stadttempel in der Wiener Seitenstettengasse Ziel eines Terroranschlags, verübt von zwei Palästinensern. Dabei kamen zwei Personen ums Leben und zahlreiche andere wurden schwer verletzt. Schon zwei Jahre zuvor war die Synagoge Ziel eines Sprengstoffattentats geworden. ${ }^{8}$ Am 27. Dezember 1985 wurden die Schalter der israelischen Fluglinie EL AL an den Flughäfen Wien und Rom die Ziele tödlicher Angriffe. Insgesamt ermordeten Terroristen dabei 19 Personen. ${ }^{9}$

Auch Neonazis und Rechtsextremisten verübten immer wieder verheerende Anschläge auf jüdische Einzelpersonen und Einrichtungen. Im Dezember 1980 wurden der Rabbiner Shlomo Lewin und seine Lebensgefährtin in ihrer Privatwohnung in Erlangen von einem deutschen Neonazi ermordet. ${ }^{10}$ Neonazis und Rechtsextreme waren insbesondere in den 1990er und 2000er-Jahren für zahlreiche Mord-, Brand- und Sprengstoffanschläge auf Synagogen, jüdische Einrichtungen und Einzelpersonen verantwortlich. Teilweise konnten Anschläge kurz vor der Ausführung noch verhindert werden, wie ein geplanter Bombenanschlag von Neonazis anlässlich der Grundsteinlegung des neuen jüdischen Gemeindezentrums in München im November 2003. ${ }^{11}$

Diese Aufzählung antisemitischer Gewalttaten ist alles andere als erschöpfend - von ungezählten antisemitisch motivierten Übergriffen, Friedhofschändungen und Sachbeschädigungen an jüdischen Einrichtungen ganz zu schweigen. Sie verdeutlicht allerdings, dass der Antisemitismus auch nach 1945 eine blutige Spur nach sich zog und

${ }^{6}$ Der Generalbundesanwalt beim Bundesgerichtshof, Ermittlungen wegen des Brandanschlags auf die Israelitische Kultusgemeinde am 13. Februar 1970 in München mangels weiterer erfolgversprechender Ermittlungsansätze eingestellt, 23.11.2017,

[https://www.generalbundesanwalt.de/SharedDocs/Pressemitteilungen/DE/2017/Pressemitteilung-vom-23-11-

2017.html], eingesehen 1.2.2020.

7 Margit Reiter, Unter Antisemitismusverdacht. Die österreichische Linke und Israel nach der Shoah, Innsbruck 2001, S. 252-260.

8 Ursula Mindler-Steiner, Ermordung von Heinz Nittel - Anschlag auf den Stadttempel, o. D., [https://www.hdgoe.at/ermordung-nittel-anschlag-stadttempel], eingesehen 1.2.2020.

9 Lisa-Sophie Heinrich, Als der Terror nach Wien Schwechat kam, in: Die Presse, 26.12.2015, [https://www.diepresse.com/4892554/als-der-terror-nach-wien-schwechat-kam], eingesehen 1.2.2020.

10 Matthias Quent/Jan Rathje, Von den Turner Diaries über Breivik bis zum NSU: Antisemitismus und rechter Terrorismus, in: Samuel Salzborn (Hrsg.), Antisemitismus seit 9/11. Ereignisse, Debatten, Kontroversen, BadenBaden 2019, S. 165-178.

${ }^{11}$ Neonazis wollten jüdisches Zentrum sprengen, in: Süddeutsche Zeitung, 12.9.2003. 
weiterhin zieht. Zusammen mit den jüngsten Anschlägen der letzten Jahre ergibt sich ein Bild ungebrochener antisemitischer Gewalttaten - auch nach und trotz der militärischen Niederlage des Nationalsozialismus und seiner Verbündeten 1945. Womit die noch immer nachhallende Erzählung, der auf Vernichtung zielende Antisemitismus sei mitsamt dem Deutschen Reich 1945 untergegangen, bereits eindrücklich widerlegt ist. Die gesellschaftspolitische Transformation hin $\mathrm{zu}$ Liberaldemokratien westlichen Musters, die nicht nur postnazistische Gesellschaften wie Deutschland und Österreich kennzeichnet, führte offensichtlich nicht zum allmählichen Verschwinden des Antisemitismus. Bemerkenswert und erschütternd an den antisemitischen Gewalttaten ist nicht nur ihre Brutalität und ihre regelmäßige Wiederkehr, sondern auch die heterogene Täterschaft, die wiederum die soziale Streuung antisemitischer Einstellungen quer durch alle Schichten und Milieus spiegelt.

\section{Externalisierung und Universalisierung}

Die damals noch frischen Eindrücke der Anschlagsserie auf jüdische Einrichtungen sowie zahlreiche antisemitische Manifestationen auf Demonstrationen im Zuge des Gaza-Konflikts $2014^{12}$ haben uns im Frühjahr 2015 dazu bewogen, eine Tagung an der Universität Innsbruck zu Ausprägungen des gegenwärtigen Antisemitismus zu initiieren. ${ }^{13}$ Neben der auch heute noch gültigen Feststellung, dass der zeitgenössische Antisemitismus, trotz seiner verheerenden Auswirkungen, nicht die nötige Aufmerksamkeit in den Geistes-, Sozial- und Kulturwissenschaften erfährt, waren es auch öffentliche Reaktionen auf die Anschläge, die uns zu diesem Vorhaben motiviert hatten. Neben vereinzelten differenzierten und kritischen Beiträgen, schien die sich anschließende Debatte zugleich durch Externalisierung und - nur auf den ersten Blick gegenläufig, aber letztlich hiermit verwandt - Universalisierung der antisemitischen Gewalttaten geprägt.

Die Externalisierung folgt einem vertrauten Muster: während die Täter symbolisch ausgebürgert werden, entledigt man sich zugleich des Antisemitismus. Dieser sei folglich ein von außen nach Europa hereingetragenes Problem, bzw. ein Exportprodukt aus der islamischen Welt oder immer nur der Antisemitismus der Anderen. Die Rede vom „importierten Antisemitismus“ hat seither eine gewisse Konjunktur, insbesondere bei

12 Julia Edthofer, Debates on Islamized Antisemitism in Austria in the Wake of the Israel-Gaza Conflict 2014 (Kantor Center Position Papers), Tel Aviv 2016,

[https://en-humanities.tau.ac.il/sites/humanities_en.tau.ac.il/files/media_server/0001/Edthofer.pdf], eingesehen 1.2.2020.

13 Die Tagung trug den Titel „,Neuer) Antisemitismus in der Gegenwartsgesellschaft“ und fand am 19. Oktober 2015 an der Universität Innsbruck statt. Eine zweite Tagung mit dem Titel „Antisemitismus in der Gegenwartsgesellschaft“ fand im Oktober 2016 ebenfalls an der Universität Innsbruck statt. 
konservativen und rechtspopulistischen Politikern und Medien. ${ }^{14}$ Auf die Spitze trieb diese Behauptung 2019 der ungarische Premierminister Viktor Orbán, der seine restriktive Migrationspolitik als probates Mittel zum Schutz der jüdischen Gemeinschaft und gegen den ,importierten Antisemitismus aus der muslimischen Welt ${ }^{* 15}$ darstellte. Wiewohl er seit einigen Jahren selbst eine antisemitische Kampagne gegen den Philanthropen und Universitätsgründer George Soros führt, ${ }^{16}$ externalisierte er die antisemitische Gefahr mit den Worten: „Die jüdische Gemeinschaft lebt hier vielleicht am sichersten in ganz Europa. Das hängt auch damit zusammen, dass es hier keine großen muslimischen Gemeinschaften gibt und damit, dass die Regierung die jüdische Gemeinschaft entschlossen schützt.“"17

Teilweise führt diese Externalisierungsstrategie gar zur Leugnung des antisemitischen Gehalts von Gewalttaten: so zeigte sich ein deutsches Gericht im Februar 2015, das einen Brandanschlag auf die Wuppertaler Synagoge verhandelte, unfähig das Offensichtliche überhaupt zu erkennen. Stattdessen folgte das Gericht der Darstellung der angeklagten jungen Männer, konnte keine „Anhaltspunkte für eine antisemitische Tat" erkennen und argumentierte in der Urteilsbegründung diese hätten lediglich die „Aufmerksamkeit auf den Gaza-Konflikt lenken wollen“ “. ${ }^{18}$ Europa avanciert in solchen Deutungsmustern zum Nebenschauplatz fremder Auseinandersetzungen, der antisemitische Gehalt wird relativiert oder gar geleugnet und in eine begründete Aversion gegen den jüdischen Staat und seine vermeintlichen Statthalter in Europa verkehrt. ${ }^{19}$

Diese Externalisierung basiert auf der Vorstellung einer vom Antisemitismus geläuterten Gesellschaft und erhält das damit verbundene Selbstbild im Sinne einer sich selbsterfüllenden Prophezeiung zugleich am Leben. Neben dem Antisemitismus der Anderen kann auch die Rede vom Antisemitismus der „Ewiggestrigen“ eine solche Funktion übernehmen und als Versuch seiner scheinbaren Bannung in der Vergangenheit begriffen werden. Im Zentrum steht jeweils die Vorstellung, dass die demokratische Nachkriegsgesellschaft den Antisemitismus überwunden hat. Verbindungslinien, die

14 So behauptete etwa Anfang 2020 der österreichische Nationalratspräsident Wolfgang Sobotka in Österreich sei ein „starker, importierter Antisemitismus' zu beobachten“. Sobotka, Noch entschiedener gegen Antisemitismus vorgehen, in: katholisch.at, 29.1.2020, [https://www.katholisch.at/aktuelles/128662/sobotka-noch-entschiedener-gegenantisemitismus-vorgehen], eingesehen 1.6.2020.

15 Importierter Antisemitismus aus der muslimischen Welt, in: kath.net, 25.5.2019, [http://www.kath.net/news/68044], eingesehen 23.6.2019.

16 Roland Clark/Nikolaus Hagen, Kalergi Plan: The Undying „White Genocide” Conspiracy Theory, in: Rantt Media, 2.5.2020, [https://rantt.com/the-kalergi-plan-explained], eingesehen 4.6.2020.

17 Zit. n. Importierter Antisemitismus, in: kath.net, 25.5.2019.

18 Deniz Yücel, Antisemitismus? Ist abgeschafft, in: taz.de, 18.2.2015, [https://taz.de/Kolumne-Besser/!5019881], eingesehen 3.7.2020.

19 Diese Form der Externalisierung hat eine lange Tradition. Über die bundesdeutsche Reaktion auf das OlympiaAttentat 1972 schreibt Eva Oberloskamp: „Die Geiselnahme der israelischen Mannschaft wurde als rein palästinensische Form des Terrorismus perzipiert, die man von der Bundesrepublik auf Dauer fern halten wollte. [...] Dem entsprach die Einschätzung, dass die Bundesrepublik [...] als ,unschuldiges Opfer ${ }^{\star}$ in den Nahostkonflikt hineingezogen worden sei, für den sie keine Verantwortung trage.“ Overloskamp, Olympia-Attentat, S. 329-330. 
dieses geläuterte Selbstbild in Frage stellen und brüchig werden lassen, werden gekappt. Das führt im Extremfall sogar dazu, dass zeitgenössische Formen des Antisemitismus überhaupt gar nicht mehr als Antisemitismus erkannt werden.

Nachdem die Journalistin Jutta Ditfurth einen der Chefideologen der Neuen Rechten und Herausgeber des verschwörungstheoretischen Compact-Magąins, Jürgen Elsässer, als Antisemiten bezeichnet hatte, klagte dieser auf Unterlassung und bekam hierbei richterlichen Beistand. In einem Verfahren am Münchener Landgericht schloss die Richterin, „dass gerade vor dem Hintergrund der Verbrechen der Nazidiktatur sowie des Holocaust die Bezeichnung, glühender Antisemit ${ }^{\prime}$ in besonderer Weise geeignet ist, den so Bezeichneten herabzuwürdigen und in seiner Ehre zu verletzen. ${ }^{\text {20 }}$ In der richterlichen Position, dass die Bezeichnung Antisemit nur auf den Anwendung finden kann, der „die Überzeugungen teilt, die zu der Ermordung von 6 Millionen Juden unter der nationalsozialistischen Schreckensherrschaft geführt haben",21 artikuliert sich ein Unvermögen die Metamorphosen und Formwandlungen des Antisemitismus auch nur ansatzweise anzuerkennen.

Die Externalisierung des Antisemitismus funktioniert gut, weil sie Anknüpfungspunkte an unterschiedlichste Einstellungsmuster und Weltbilder bietet. Die gesellschaftliche „Mitte“ kann sich ihrer selbst versichern und den Antisemitismus wahlweise an Rändern und Extremen, bei Außenstehenden und Anderen verorten. Für die politische Rechte ist Antisemitismus neuerdings überhaupt eine Folge der Migration und werde entsprechend durch Einwanderungsstopp und Abschiebungen bekämpft. ${ }^{22}$ Während weite Teile der Linken insbesondere den israelbezogenen Antisemitismus - sofern der antisemitische Gehalt nicht überhaupt abgestritten wird - ohnehin als verständliche Reaktion auf Imperialismus und Kolonialismus rationalisieren. ${ }^{23}$ Sie verdrängen in diesem Zuge nicht nur die antikoloniale Entstehungsgeschichte des jüdischen Staates, sondern beschuldigen diesen gar als imperialistischen Brückenkopf und koloniales Übel.

Zweifelsohne hat in den letzten Jahren insbesondere die Deutung vom „importierten Antisemitismus“ - oder auch vom „Antisemitismus der Migranten“ - beinahe hegemonialen Charakter im öffentlichen Diskurs eingenommen. Der Antisemitismus wird auf diese Weise zwar nicht per se negiert, aber eben einer bestimmten Bevölkerungsgruppe überantwortet. Die kurzfristigen Vorteile dieser Verschiebung sind evident: Sie entlasten weite Teile der Gesellschaft nicht einfach nur, sondern sie kitten

${ }^{20}$ Landgericht München, Urteil vom 10.12.2014, 25 014197/14,

[https://www.juris.de/jportal/prev/JURE170032899], eingesehen 3.7.2020.

21 Ebd.

22 Siehe exemplarisch Alexander Häusler, Feindbild Moslem. Türöffner von Rechtsaußen hinein in die Mitte?, in: Gideon Botsch u. a. (Hrsg.), Islamophobie und Antisemitismus - ein umstrittener Vergleich, Berlin 2012, S. 169-190, hier S. 174 .

${ }^{23}$ Siehe exemplarisch Julia Edthofer, Vom antiimperialistischen Antizionismus zur aktuellen Boykottbewegung, in: Österreichische Zeitschrift für Soziologie 42 (2017), S. 407-424. 
zudem die Brüche im anti-antisemitischen Tabu der postnazistischen Gesellschaft ${ }^{24}$ und verhindern schmerzhafte (Selbst-) Reflexion.

In diesen Wirkungszusammenhang ist auch der Mechanismus der Universalisierung antisemitischer Gewalttaten einzuordnen. Indem die Anschläge auf jüdische Einrichtungen als Teil eines Kulturkampfes des Islamismus oder des Islam gegen den (jüdisch-)christlichen Westen verallgemeinert werden, verliert sich auch in diesem Deutungsmuster der spezifische antisemitische Gehalt solcher Gewaltsituationen. Einerseits ist diese Deutung nicht gänzlich von der Hand zu weisen, schließlich ist es geradezu Kernelement des Antisemitismus, dass dieser Jüdinnen und Juden als die Agenten gesellschaftlicher Modernisierungsprozesse und damit einhergehender Verwerfungen imaginiert und ins Visier nimmt. Dass Jüdinnen und Juden und insbesondere der jüdische Staat in der Ideologie diverser islamistischer Gruppierungen nicht einfach nur als Stellvertreter, sondern gar als heimliche Strippenzieher hinter den Vereinigten Staaten oder gleich dem gesamten Westen firmieren, steht genau in dieser antisemitischen Tradition. Andererseits wird diese Deutung dann problematisch, wenn der Antisemitismus im Narrativ vom allumfassenden Kulturkampf verschwindet.

Auf diese Weise wird der Antisemitismus universalisiert: „Jede Form des Antisemitismus", um eine Redewendung des deutschen Justizministers Heiko Maas aufzugreifen, wird dann zu einem „Angriff auf uns alle“. ${ }^{25}$ Diese Rhetorik unterschlägt aber das Wesentliche antisemitischer Gewalttaten, die eben nicht wahllos gegen „uns alle" gerichtet sind, sondern gegen Jüdinnen und Juden und - den Spuren des antisemitischen Wahns folgend - gegen deren „Kollaborateure“. ${ }^{26}$ Wird die Szenerie zu einem „Angriff auf uns alle“ verallgemeinert, dann ist am Ende möglicherweise gar nichts mehr zu erkennen: Als im November 2015 ein äußerst opferreicher Anschlag auf die Pariser Konzerthalle „Bataclan“ verübt wurde, spielte die offenkundige antisemitische Motivation der Täter im öffentlichen Diskurs kaum mehr eine Rolle - obwohl bekannt war, dass das Veranstaltungslokal wiederholt Gegenstand antisemitischer Schmähungen und Drohungen war. ${ }^{27}$

Neben diese beiden Mechanismen tritt in der politischen Arena eine problematische Pattstellung: Teile der politischen Rechten und der Linken schieben sich den Antisemitismus gegenseitig in die Schuhe, projizieren ihn auf andere oder leugnen ihn aus strategischen Gründen wahlweise. ${ }^{28}$ Letztlich führt das dazu, dass nicht nur der

24 Vgl. Juliane Wetzel, Judenfeindliche Stereotypisierungen: Das Beschneidungsurteil im öffentlichen Diskurs, in: Johannes Heil/Stephan J. Kramer (Hrsg.), Beschneidung: Das Zeichen des Bundes in der Kritik. Zur Debatte um das Kölner Urteil, Berlin 2012, S. 264-275, hier S. 265.

25 Heiko Maas, Jede Form von Antisemitismus, in: twitter.com, 10.12.2017,

[https://twitter.com/HeikoMaas/status/939985057174949888], eingesehen 9.7.2020.

${ }^{26}$ Siehe zur Logik des antisemitischen Selbstmordattentats insbesondere Gerhard Scheit, Suicide Attack. Zur Kritik der politischen Gewalt, Freiburg i. Br. 2004.

27 Alex Feuerherdt, Bataclan. Ein antisemitischer Anschlag, in: mena-watch.com, 17.11.2015, [https://www.menawatch.com/bataclan-ein-antisemitischer-anschlag], eingesehen 9.7.2020.

${ }^{28}$ Siehe dazu auch Julia Edthofer, Rassismus- oder Antisemitismuskritik? Eine antirassistische Perspektive auf den 
Antisemitismus der Mitte vollkommen aus dem Blick gerät, sondern darüber hinaus auch jener im eigenen politischen Lager.

\section{Antisemitismus in der Migrationsgesellschaft?}

2015 hatten wir für die erste unserer Tagungen ursprünglich den Titel „Antisemitismus in der Migrationsgesellschaft" ins Auge gefasst. Wir wollten damit der Tatsache Rechnung tragen, dass die mitteleuropäischen Gesellschaften in erheblichem Maße von und durch Migration geprägt sind. Einer OECD-Statistik zufolge waren mit Stand 2018 beinahe ein Drittel der schweizerischen, 19 Prozent der österreichischen und 16 Prozent der deutschen Wohnbevölkerung im Ausland zur Welt gekommen. Damit übertrifft die Schweiz sogar „klassische Einwanderungsländer“ wie Australien, Neuseeland, Kanada oder die Vereinigten Staaten in erheblichem Maße. Auch Österreich und Deutschland weisen nach dieser Erhebung Spitzenplätze noch vor den Vereinigten Staaten oder dem Vereinigten Königreich auf. ${ }^{29}$ Selbst unter Anwendung einer sehr engen Definition, wenn also beide Elternteile im Ausland zur Welt kamen, besitzt ein Viertel der österreichischen Bevölkerung einen sogenannten Migrationshintergrund. ${ }^{30}$

Mobilitäts- und Migrationserfahrungen bestimmen in zunehmendem Maße die Biografien weiter Teile der Bevölkerung. Hierin spiegelt sich nicht zuletzt die politische Förderung von grenzüberschreitender Mobilität, die aber vor allem dann als opportun gilt, sofern sie notwendiger Bestandteil von Bildungs- und Berufskarrieren ist. Trotz unbestreitbarer Migrationsrealitäten ist der Begriff „Migrationsgesellschaft“ allerdings bis heute politisch umstritten. Noch immer weigern sich maßgebliche politische Kräfte sowohl in Deutschland als auch in Österreich dies anzuerkennen. Wir halten den Begriff „Migrationsgesellschaft" demgegenüber für eine neutrale Tatsachenbeschreibung, die nicht zuletzt die soziale Wirklichkeit in den urbanen Ballungszentren beschreibt. In Wien, um eine der größten deutschsprachigen Städte beispielhaft heranzuziehen, waren Anfang 2018 laut amtlicher Statistik 41 Prozent der Bevölkerung im Ausland geboren oder besaßen eine andere als die österreichische Staatsbürgerschaft. ${ }^{31}$ Rechnet man die zweite bereits im Inland geborene Generation mit österreichischer Staatsbürgerschaft hinzu, so

Zusammenhang von Islamismus und Antisemitismus, 20.6.2015,

[https:// forschungsgruppefipu.wordpress.com/2015/06/20/gastbeitrag-rassismus-oder-antisemitismuskritik-julia-

edthofer/], eingesehen 1.2.2020.

${ }^{29}$ OECD Databases on Migration, o. D.,

[https://www.oecd.org/migration/mig/oecdmigrationdatabases.htm], eingesehen 1.6.2020.

30 Statistik Austria, Bevölkerung mit Migrationshintergrund im Überblick (Jahresdurchschnitt 2019), 18.3.2020,

[https://www.statistik.at/wcm/idc/idcplg?IdcService=GET_PDF_FILE\&RevisionSelectionMethod=LatestRelease $\mathrm{d} \& \mathrm{dD}$ ocName$=033240$ ], eingesehen 1.6.2020.

31 Stadt Wien, Daten und Fakten zur Migration 2019 - Wiener Bevölkerung, o. D.,

[https://www.wien.gv.at/menschen/integration/daten-fakten/bevoelkerung-migration.html], eingesehen 1.6.2020. 
kann man davon ausgehen, dass die absolute Mehrheit der Wiener Bevölkerung über familiäre Migrationserfahrung verfügt.

Die ideologische Fiktion eines homogenen Nationalstaates mit einem gemeinsamen „Geschichtsbewusstsein“ wird angesichts dieser Faktenlage immer brüchiger. ${ }^{32}$ Auf diesen Sachverhalt hätte insbesondere auch das Bildungssystem Rücksicht zu nehmen. Denn eine solche Fiktion und die mit ihr verbundenen Mythen sind keine adäquate Grundlage für die Analyse und Bekämpfung des Antisemitismus. Die vorherrschende Geschichtspolitik der letzten Jahrzehnte - die auch die Grundlage für die historischpolitische Bildungsarbeit geliefert hat - hat aber insbesondere in Deutschland und Österreich genau das Gegenteil getan. Zwar wird vordergründig häufig auf die Geschichte des Nationalsozialismus und den Holocaust rekurriert, beide Gesellschaften wurden und werden allerdings auf je unterschiedliche Weise als Opfer des Nationalsozialismus gedeutet. ${ }^{33}$ Als in Deutschland in den 1990ern ein nationaler Lernprozess („Geschichtsaufarbeitung“仿) einsetzte, kopierte man in Österreich mit einigen Jahren Verzug auch dieses geschichtspolitische Modell. Wie dieses geschichtspolitische Erfolgsmodell funktioniert, formulierte der ehemalige deutsche Bundeskanzler Gerhard Schröder vor 15 Jahren mit der Formel einer besonderen Verantwortung der Deutschen vor der Geschichte und einer hieraus resultierenden „moralischen Verpflichtung“, die zu einem „Teil unserer nationalen Identität“ geworden ist. ${ }^{34}$ Mit der Integration des Nationalsozialismus in das nationale Selbstbild und der Übernahme dieser besonderen Aufgabe befreit sich die Nation zugleich und implizit vom (eigenen) Antisemitismus. ${ }^{35}$

Jede Geschichtserzählung und Erinnerung stiftet im Sinne eines „Bindemittels“36 soziale Kohäsion und übernimmt damit eine integrierende Funktion. Einen Störfaktor in diesem Selbstbild von einer Gesellschaft, die aus dem Nationalsozialismus gelernt habe, stellen nicht erst seit heute Migranten und Migrantinnen dar. Deren Ausschluss ist bereits in den historischen Erzählungen entlang der Achse eines nationalen Narratives angelegt. Insofern ist es auch nicht überraschend, dass die ohnehin überforderten Bildungsinstitutionen im Angesicht von Migration beunruhigt sind und Lehrer und Lehrerinnen sich beklagen, dass sich (post-)migrantische Schüler und Schülerinnen nicht in die etablierte national-kulturelle Lernkultur, die ein Lernen aus Verantwortung

32 Dirk Rupnow, Deprovincializing Contemporary Austrian History. Plädoyer für eine transnationale Geschichte Österreichs als Migrationsgesellschaft, in: Zeitgeschicbte 40 (2013), Heft 1, S. 6-21.

33 In Österreich hallte lange die Vorstellung nach, das erste Opfer der deutschen NS-Aggression gewesen zu sein („Opferthese“). Zu Deutschland siehe Hannes Heer, Hitler war's. Die Befreiung der Deutschen von ihrer Vergangenheit, Berlin 2005.

34 Gerhard Schröder, „Wir stehen erst jetzt am Ende einer langen Nachkriegszeit“, in: Süddeutscher Zeitung, 7.5.2005.

35 „Wir wollen und wir werden nicht zulassen, dass Unrecht und Gewalt, dass Antisemitismus, Rassismus und Fremdenfeindlichkeit wieder eine Chance bekommen." (Ebd.)

${ }^{36}$ Carola Sachse/Edgar Wolfrum, Stürzende Denkmäler. Nationale Selbstbilder postdiktatorischer Gesellschaften in Europa - Einleitung, in: Carola Sachse/Edgar Wolfrum/Regina Fritz (Hrsg.), Nationen und ihre Selbstbilder. Postdiktatorische Gesellschaften in Europa, Göttingen 2008, S. 7-35, hier S. 13. 
gegenüber der „eigenen“ Geschichte postuliert, ${ }^{37}$ einfügen ließen. Und tatsächlich hat diese Feststellung - die realiter Symptom pädagogischer Unfähigkeit ist - letztlich ihr Wahres an sich: tatsächlich wurden und werden Migranten und Migrantinnen aus dieser Geschichte hinausgeschrieben. Erst langsam findet hier in den letzten Jahren ein Umdenken statt: der Inklusion von Migrationsgeschichte in die Geschichtsbücher müsste aber längst auch eine entsprechende Pädagogik folgen, die das Lernen aus der Geschichte - im Sinne von Verantwortung statt Identität - gerade nicht von einer spezifischen nationalen Herkunft abhängig macht.

Wir haben uns 2015 nach einigen Debatten letztlich für den Tagungstitel „Antisemitismus in der Gegenwartsgesellschaft“ entschieden und diesen auch für die Folgekonferenz 2016 verwendet. In der Zwischenzeit hatte sich nämlich ein Massenfluchtereignis ereignet, das eine neutrale Verwendung des Begriffs „Migrationsgesellschaft" beinahe unmöglich machte. Wir wollten in der Hitze der Debatten im Sommer 2015 den Eindruck vermeiden, vom „Antisemitismus der Anderen“ zu sprechen. Und tatsächlich stehen sich seit damals Proponenten einer „Willkommenskultur“ und Gegner jeder Einwanderung beinahe unversöhnlich gegenüber. Politisch haben letztere in vielen europäischen Ländern die Oberhand gewonnen: Seit Sommer 2015 sind zahlreiche europäische Binnengrenzen geschlossen oder erschwert passierbar, das Schengener Abkommen ist de facto ausgesetzt und die europäischen Außengrenzen, insbesondere jene im Mittelmeer, haben sich in Massengräber verwandelt. Der islamistische Terror wurde damit keineswegs gebannt, schließlich handelte sich bei den Attentätern von Brüssel, Kopenhagen oder Paris, die eingangs erwähnt wurden, nicht um Einwanderer, sondern um Personen, die in Europa zur Welt gekommen waren.

Die Mehrheitsbevölkerung schielt dennoch nach wie vor beinahe exklusiv und obsessiv auf den „Antisemitismus der Migranten“ und in der Zwischenzeit hat rechtsextremer Antisemitismus Fahrwasser bekommen. Das ist nur scheinbar ein Paradoxon. In Zeiten gesellschaftlicher Verwerfungen hat, wie die europäische Geschichte beweist, auch der Antisemitismus mit seiner ungebrochenen psychischen Attraktivität stets Konjunktur. Unter dem Schlagwort „Der große Austausch“ kultivieren rechte Agitatoren unterschiedlicher Couleur äußerst erfolgreich einen alten Verschwörungsmythos in modernisierter Form: Jüdinnen und Juden und ihre Verbündeten würden heimlich die Migrationsströme nach Europa lenken, um die ,autochthone“ europäische Bevölkerung zu ersetzen. ${ }^{38}$ Diese Erzählung ist letztlich ein Wiedergänger alter antisemitischer Mythen über einen jüdischen Plan zur Zerstörung Europas. Sie hat über den bereits erwähnten Viktor Orbán ihren Weg in den politischen Mainstream gefunden und wird auch von

${ }^{37}$ Astrid Messerschmidt, Geschichtsbewusstsein ohne Identitätsbesetzungen - kritische Gedenkstättenpädagogik in der Migrationsgesellschaft, in: bpb.de, 14.1.2016, [https://www.bpb.de/apuz/218720/kritischegedenkstaettenpaedagogik-in-der-migrationsgesellschaft?p=all], eingesehen 14.7.2020.

38 Clark/Hagen, Kalergi-Plan; A. Dirk Moses, „White Genocide“ and the Ethics of Public Analysis, in: Journal of Genocide Research 21 (2019), Heft 2, S. 201-213. 
Exponenten der FPÖ oder AfD regelmäßig aufgegriffen. Erst kürzlich glaubte die AfDAbgeordnete Beatrix von Storch die geheimen „Pläne für einen Massenaustausch“ gefunden $\mathrm{zu}$ haben, tatsächlich handelte sich um eine banale soziodemographische Studie. ${ }^{39}$

So absurd dieser Mythos sich auch anhört, so gefährlich und entschlossen sind seine Anhänger. In Kalifornien stürmte im April 2019 ein schwerbewaffneter 19-jähriger Täter namens John T. Earnest die Chabad-Synagoge in Poway, einer Vorstadt von San Diego. Er eröffnete das Feuer auf die dort versammelte jüdische Gemeinde, die gerade den letzten Feiertag des Pessachfests beging, und tötete eine Frau. ${ }^{40}$ Das erklärte Vorbild von Earnest war Brenton Harrison Tarrant, ein 28-jähriger Australier, der im Monat zuvor 51 Gläubige in einer Moschee im neuseeländischen Christchurch brutal massakriert hatte. Sowohl Earnest als auch Tarrant zeigten sich als überzeugte Anhänger des Verschwörungsmythos vom ,großen Austausch“, der Vorstellung nichteuropäische Völker wollten „die weiße Rasse“ vernichten. Nur geographisch weit von Europa entfernt wiesen diese Taten unmittelbare Bezüge mitten nach Europa auf: Tarrant korrespondierte und traf sich in Wien mit Vertretern der rechtsextremen „Identitären Bewegung Österreich" und spendete an deren Rädelsführer, Martin Sellner, wie später bekannt geworden ist, 1.500 Euro. ${ }^{41}$ Nur ein halbes Jahr nach den Anschlägen von Christchurch und Poway, im Oktober 2019, ermordete der deutsche Rechtsextremist Stephan Balliet, beim Versuch am jüdischen Feiertag Jom Kippur in die Synagoge in Halle einzudringen, eine Passantin und anschließend den Gast eines türkischen Imbisslokals. Auch Balliet bezog sich auf Tarrant und erklärte im Nachgang, so hieß es in Medienberichten, dass er bereue, „keine jüdischen Menschen und überhaupt nur so wenige Personen getötet zu haben" ${ }^{42}$ Zuletzt ermordete Tobias Rathjen, ein rechtsextremer Verschwörungstheoretiker, im Februar 2020 zehn Menschen im hessischen Hanau. Als Opfer wählte er gezielt Menschen aus, die er als Migranten zu erkennen glaubte. In einem Manifest, das er im Internet veröffentlichte, äußerte er genozidale Phantasien und rief unter anderem zur Vernichtung sämtlicher arabischer Staaten sowie Israels auf. ${ }^{43}$

39 Patrick Gensing, Legenden und Mythen. Keine Verschwörung ohne „Geheimplan“, in: tagesschau.de, 12.5.2020, [https://www.tagesschau.de/faktenfinder/verschwoerungen-geheimplaene-101.html], eingesehen 10.7.2020.

40 Earnest kündigte seine Tat in einem online veröffentlichten „Manifest“ an. Der Text findet sich online unter [https://archive.org/details/john-t-ernest-manifesto-8chan-pol-april-27-2019-an-open-letter], eingesehen 1.2.2020.

${ }_{41}$ Dokumentationsarchiv des österreichischen Widerstands, Die „Identitäre Bewegung Österreich“ nach Christchurch. Neues von ganz rechts, März 2019, [https://www.doew.at/erkennen/rechtsextremismus/neues-von-ganzrechts/archiv/maerz-2019/die-identitaere-bewegung-oesterreich-nach-christchurch], eingesehen 1.2.2020.

42 Geständnis über Anschlag von Halle, in: Deutsche Welle, 30.3.2020, [https://www.dw.com/de/berichtgest $\%$ C3\%A4ndnis- $\%$ C3\%BCber-anschlag-von-halle/a-52955191], eingesehen 1.4.2020.

43 Frank Jansen/Sven Lemkemeyer, Was über den Täter von Hanau bekannt ist, in: Der Tagesspiegel, 21.2.2020, [https://www.tagesspiegel.de/politik/wer-war-tobias-rathjen-was-ueber-den-taeter-von-hanau-bekannt-

ist/25566016.html], eingesehen 1.4.2020. 
Wir halten es heute für notwendiger denn je, über Antisemitismus in der Migrationsgesellschaft zu sprechen. Zur Migrationsgesellschaft gehören alle: schlechterdings auch die Antisemiten und Antisemitinnen aus unterschiedlichen politischen Lagern und der Mitte der sogenannten „Mehrheitsgesellschaft“ - und damit eben auch jene aus (post-)migrantischen Milieus. Mit dem Gesellschaftswandel der jüngeren Vergangenheit - der zwar nicht mit Migration gleichgesetzt werden kann, aber sehr wohl auch mit ihr zusammenhängt - nimmt ebenfalls der Antisemitismus neue Formen an. Der „alte“ und herkömmliche Antisemitismus ist damit nicht einfach verschwunden, sondern hat sich lediglich modernisiert. Die Gesellschaft ist in sich pluralistischer und diverser geworden, wofür auch der Begriff der Superdiversität ${ }^{44}$ geprägt wurde. Hiermit verbunden, so unsere These, sind auch die Ausdrucksformen des Antisemitismus vielgestaltiger und unübersichtlicher geworden - und damit mögliche antisemitische Bündnisse.

Scheinbar überwunden geglaubte antisemitische Verschwörungstheorien drängen heute wieder zurück in die politische Arena: die „Protokolle der Weisen von Zion“ als Erzählung vom „großen Austausch“, völkische Globalisierungs- und Kapitalismuskritik in Form von personalisierten antisemitischen Hetzkampagnen gegen George Soros und andere sogenannte „Globalisten“ und vermeintliche Strippenzieher. Neonazis und Rechtsextreme spüren in diesem Klima Aufwind und schreiten zur Tat. Auch linker Antisemitismus artikuliert sich wieder, wie jüngst das Beispiel der antisemitischen und antiisraelischen Kampagnen der britischen Labour-Parteiführung verdeutlicht, äußerst offen und unverstellt - ganz so als hätte es die innerlinke Kritik der vergangenen beiden Jahrzehnte niemals gegeben. Und nicht zuletzt hat der auch in Europa beheimatete islamistische Terrorismus, Jüdinnen und Juden sowie jüdische Einrichtungen zu bevorzugten Zielen seiner Gewaltexzesse erkoren. Diese Konstellationen erfordern zugleich ein besonnenes Augenmerk als auch entschiedenes Handeln gegen jede Form des Antisemitismus - unabhängig von strategischen Erwägungen.

${ }^{44}$ Steven Vetrovec, Super-diversity and its implications, in: Ethnic and Racial Studies 30 (2007), Heft 6, S. 1024-1054. 
